Der Hans-Janssen-Preis wurde Christine BeEse, Berlin, in Anerkennung ihrer Arbeit „Neue Horizonte im Städtebau - Die Form der Stadt bei Marcello Piacentini (1881-1960)“"verliehen.

\title{
Christine Beese Marcello Piacentini. Moderner Städtebau in Italien
}

Sehr geehrter Herr Prof. Tangermann, sehr geehrte Mitglieder der Akademie, sehr geehrte Damen und Herren, zuallererst möchte ich mich bei Ihnen nachdrücklich für die Auszeichnung meiner Arbeit mit dem Hans-Janssen-Preis 2014 bedanken. Wie Sie ja sicherlich wissen, ist die Kunstgeschichte zuweilen ein einsames Geschäft. Wochen und Monate in Bibliotheken und Archiven lassen einen ab und zu einmal daran zweifeln, ob die Welt „da draußen“ den eigenen Forschungsgegenstand für ebenso relevant, die eigene Vorgehensweise für ebenso vielversprechend hält wie man selbst. Umso mehr hat es mich gefreut, eine derartige Anerkennung von angesehenen Wissenschaftlerinnen und Wissenschaftlern aus ganz verschiedenen Disziplinen zu erfahren. Des

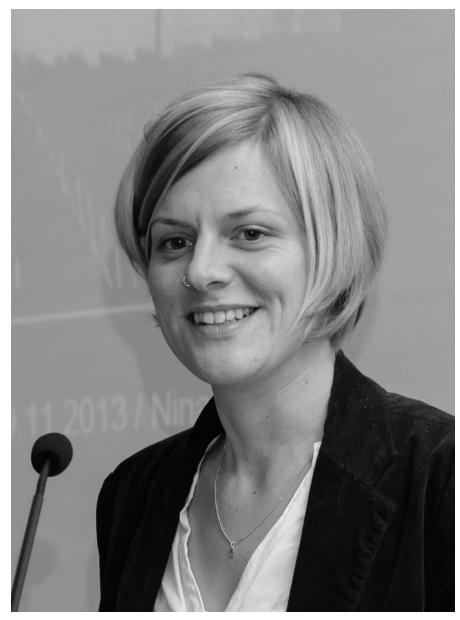

Christine Beese, wissenschaftliche Assistentin im Kunsthistorischen Institut der FU Berlin, Trägerin des Hans-Janssen-Preises 2014

Weiteren möchte ich mich bei Ihnen für die Möglichkeit bedanken, meine Arbeit, die in diesem Jahr im Reimer-Verlag erschien, in diesem Rahmen kurz vorstellen zu dürfen.

\section{Hintergrund}

Bevor ich Ihnen die Ergebnisse meiner Untersuchung schildere, möchte ich Sie zunächst mit der Figur des Architekten Marcello Piacentini vertraut machen (Abb. 1). Marcello Piacentini wurde 1881 als Sohn des renommierten Architekten Pio Piacentini in Rom geboren. Mit Ausrufung Roms zur Hauptstadt des Vereinten Königreichs Italien im Jahr 1871 hatte dort eine lebhafte Debatte um die Aufgaben und die Gestalt der neuen Kapitale begonnen. Während sich sein Vater mit architektonischen Einzelprojekten wie einem neobarocken Ausstellungspalast 
auf der Via Nazionale hervortat, entwickelte Piacentini ein größeres Interesse an der jungen Disziplin des Städtebaus. In ihr sah er die Gelegenheit, historische und moderne Architektur miteinander zu verbinden und selbst ein neues Feld zu prägen. Obschon Piacentini bereits in den 1910er Jahren unter dem sozialistischen Bürgermeister Ernesto Nathan erfolgreich war, gewann seine Karriere mit dem Aufstieg Mussolinis in den 1920er Jahren besonders an Fahrt.

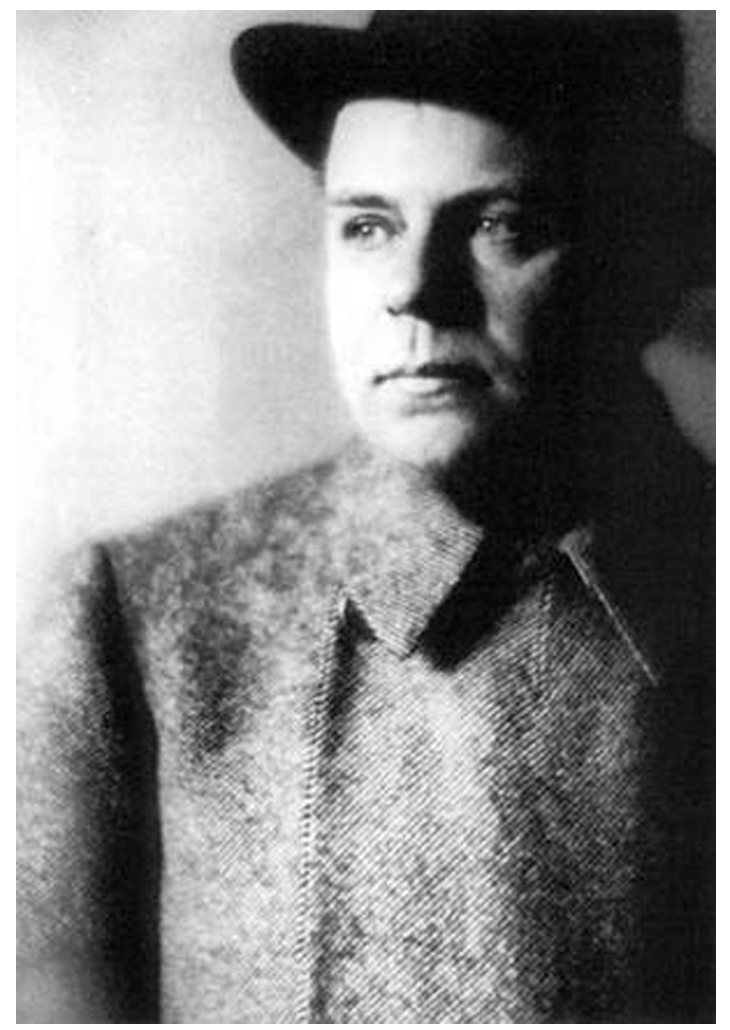

Abb. 1: Portrait von Marcello Piacentini. Aus: Mario Lupano: Marcello Piacentini, Rom 1991.

Sowohl im Rahmen staatlicher als auch im Rahmen privater Aufträge bewies er die Fähigkeit, große Aufträge für sich zu gewinnen und sie als Chefarchitekt erfolgreich umzusetzen. Dabei entwickelte er nicht nur die Masterpläne sowie Schlüsselwerke innerhalb der neuen Ensembles. Er koordinierte zugleich die Zusammenarbeit von Investoren und der Kommune, von privaten Architekten und Mitarbeitern der städtischen Baubehörden. Zwei besonders wichtige Beispiele sind in diesem Zusammenhang die Piazza della Vittoria in Brescia (Abb. 2) und die Universitätsstadt in Rom (Abb. 3). 


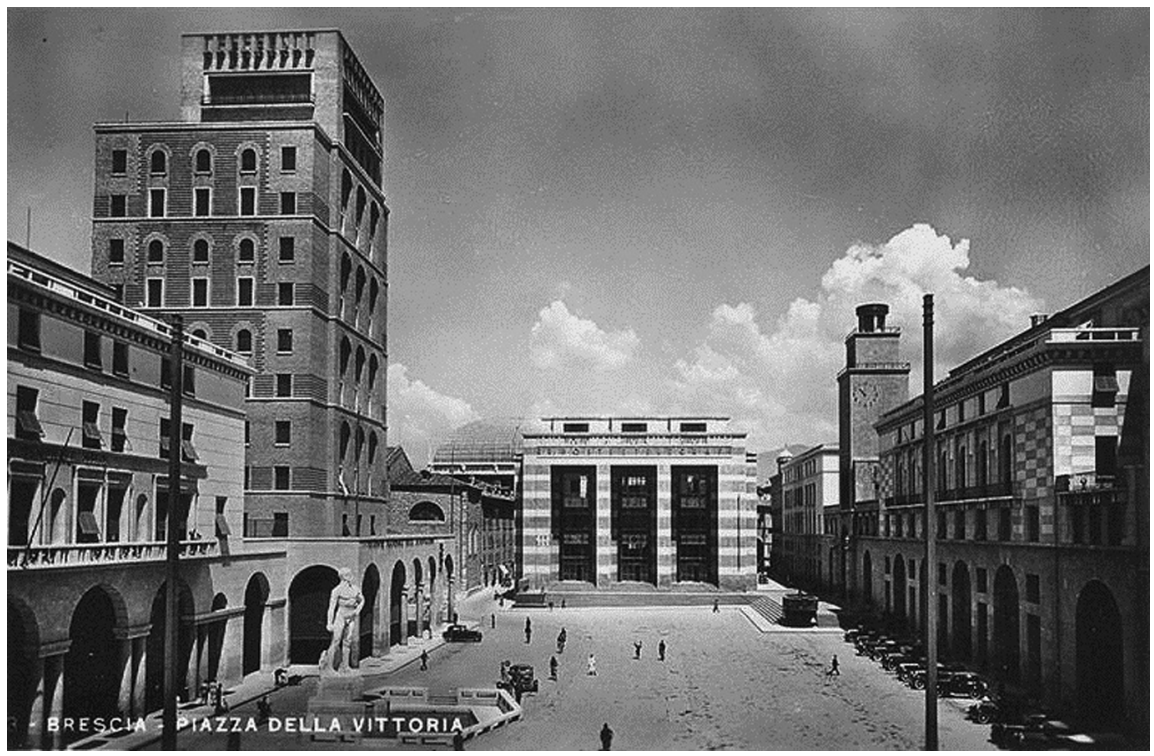

Abb. 2: M. Piacentini, Piazza della Vittoria in Brescia, 1929-1932. Aus: Beese (2015), Abb. 182.

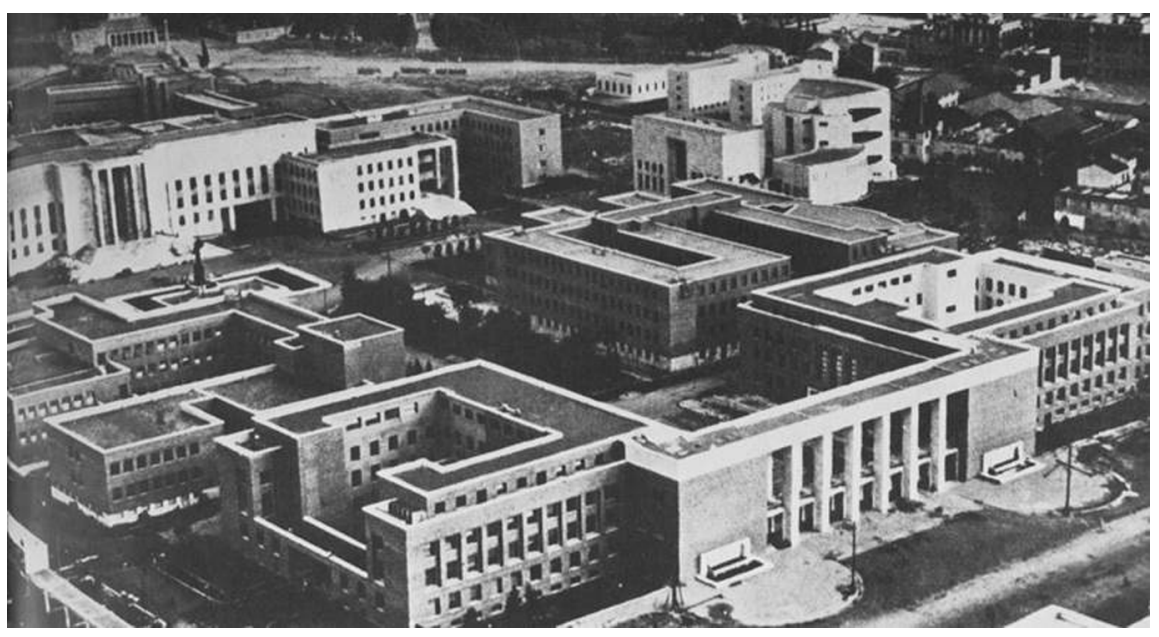

Abb. 3: Luftaufnahme der römischen Universitätsstadt von 1935. Aus: Beese (2015), Abb. 76.

Während die Neugestaltung von Brescia 1928 zunächst ein Anliegen der Kommune sowie der lokalen Industriellen war, galt die Universitätsstadt von Beginn an als Prestigeprojekt des nun etablierten faschistischen Staates. Stilistisch zeigen beide Ensembles, wie Piacentini sich vom Jugendstil seiner frühen Werke 
entfernte und eine einfachere, strengere Formensprache anstrebte, die er zu dieser Zeit bei Wilhelm Kreis in Deutschland beobachtet hatte. Eine nähere Betrachtung zeigt, dass Piacentini zwar seine Architektursprache modernisiert, im Bereich des Städtebaus jedoch an traditionell-geschlossenen Stadträumen festhält.

Musste Piacentini sich im Bereich des öffentlichen Bauens zunächst gegen den neobarocken Architekten Armando Brasini behaupten, so kam die größte Konkurrenz bald aus den Reihen der jüngeren Studenten, die besonders für die funktionalistische Architektur der klassischen Moderne eintraten. Zunächst stand Piacentini den Erneuerungstendenzen der jungen „Rationalisten“ aufgeschlossen gegenüber, nahm sie 1932 auch in sein Team zum Bau der römischen Universitätsstadt auf. Je mehr die Rationalisten jedoch für sich in Anspruch nahmen, die faschistische Staatskunst zu repräsentieren, desto mehr sah Piacentini sich gezwungen, auf klassische Formen zurückzugreifen um seinen Machtanspruch durchzusetzen.

Höhepunkt dieser Entwicklung ist der zweite Bebauungsplan für die römische Weltausstellung E 42. Hatte Piacentini noch 1937 gemeinsam mit Rationalisten wie Giuseppe Pagano an einem Masterplan für das neue faschistische Zentrum im Südosten der Stadt gearbeitet, so gelingt es ihm 1938 seinen eigenen Plan durchzusetzen. Dieser weist anstelle der Kleeblattkreuzungen, Punkthochhäuser und Hochstraßen das klassische Konzept der Platzgruppen und Prachtstraßen auf (Abb. 4). Obschon nie öffentlich mit einem solchen Amt betraut, gilt er nun als Mussolinis erster Architekt.

Im Einklang mit Mussolinis Autarkie-Politik fordert Piacentini sodann eine Rückkehr zu heimischen Formen und Materialien, zu Säulen und Bögen, zu Travertin und Marmor. Mittels zahlreicher Zeitungsartikel zeichnet er von sich das Bild eines „Verwalters der faschistischen Staatskunst“.

\section{Rezeptionsgeschichte}

Besonders unangenehm stößt es daher vielen Zeitgenossen auf, dass Piacentini nach dem Krieg ins öffentliche Leben zurückkehren, einige Projekte wie die E 42 zu Ende führen und auch die Leitung der römischen Architekturhochschule wieder übernehmen kann. Angesichts der zunehmend ablehnenden Stimmung gegen Piacentini sehen die Rationalisten nun die Gelegenheit gekommen, den alten Herrn aus der ersten Riege zu verdrängen und selbst in den Vordergrund zu treten. So als hätte es ihr Engagement für einen revolutionär-faschistischen Staatsstil nie gegeben, präsentieren sie ihre Architektur nun als antifaschistisch, als Opfer politischer Vereinnahmung. 


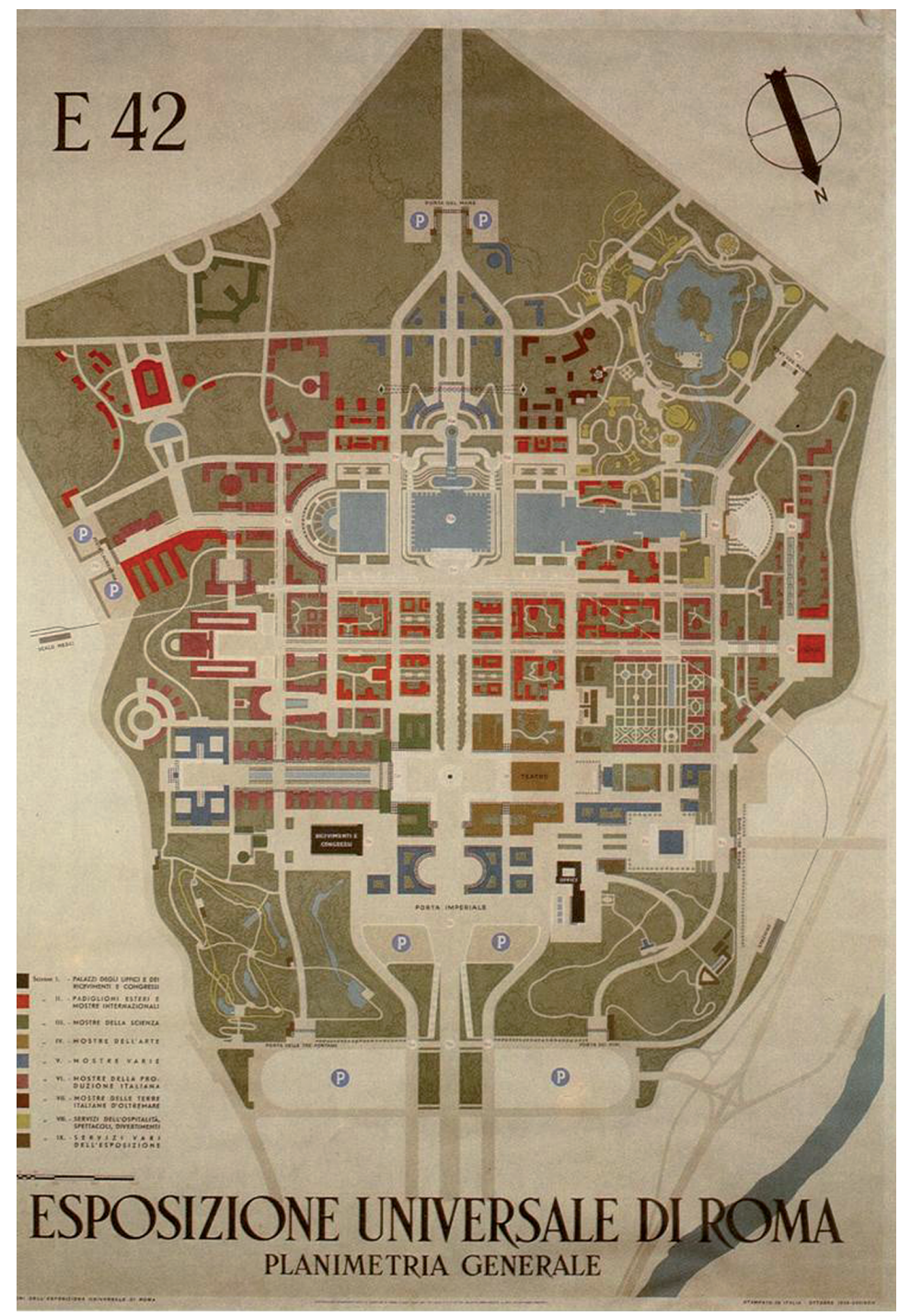

Abb. 4: M. Piacentini, G. Pagano, L. Piccinato, E. Rossi, L. Vietti, Generalplan der E 42, Oktober 1939. Aus: Maurizio Calvesi: E 42, utopia e scenario del regime, Bd. 2, Venedig 1987, S. 201.

Tatsächlich hatten einige der Rationalisten, wie Giuseppe Pagano, dem Faschismus in den 1940er Jahren enttäuscht den Rücken zugekehrt und waren als Mitglieder der Resistenza im Konzentrationslager Mauthausen ermordet worden hieraus begründeten die Rationalisten in der Nachkriegszeit ihre moralische Überlegenheit. 
Als „Dämon“ der italienischen Architekturgeschichte stigmatisiert, wurde es nach seinem Tod 1960 still um Piacentini. Eine wissenschaftliche Beschäftigung mit seinem Werk schien unangebracht, das Urteil stand fest: Piacentini sei ein reaktionärer Neoklassizist gewesen, der der Entwicklung der antifaschistischen Moderne im Weg gestanden habe. Er sei verantwortlich gewesen für all die Abrisse in historischen Stadtzentren, in deren Folge tausende mittellose Stadtbewohner in abgelegene Vororte ziehen mussten.

Nach der Veröffentlichung einer ersten Monographie von Mario Lupano im Jahr 1991 wurde bereits immer offensichtlicher, dass sich ein solch einseitiges Urteil auf Dauer nicht würde halten können. Angesehene Forscher wie Giorgio Ciucci beschäftigten sich intensiver mit verschiedenen Protagonisten und städtebaulichen Projekten des faschistischen Ventennio und legten die Vermutung nahe, dass sich das Architekturgeschehen viel pluralistischer gestaltete, als es eine Diktatur vermuten lässt. Nicht immer deckten sich die Ziele der römischen Kommune mit den Zielen der römischen Kapitale. Verschiedene Architekten warben um die Gunst Mussolinis, belieferten ihn mit diversen städtebaulichen Konzepten, die keinesfalls zu einem kohärenten Plan zusammengefasst wurden. Vielmehr nahm der „Duce“ mal diese, mal jene Anregung auf und ließ jede Gruppe auf neue Aufträge hoffen.

\section{Inhalt der Arbeit}

Was also machte Piacentinis Städtebau wirklich aus? Welche Haltung und welche Prinzipien entwickelte er im Laufe der Jahre und wie setzte er sie um? Dies waren die Fragen, die mich selbst bei meiner Untersuchung geleitet haben. Welche Ziele verfolgte er konkret, welche Kosten hat er dafür in Kauf genommen? Selbstverständlich erlaubt es die Zeit an dieser Stelle nicht, Ihnen all meine Ergebnisse detailliert vorzustellen. Die wichtigsten Aspekte möchte ich jedoch anhand meiner Gliederung erläutern (Abb. 5).

\section{Von der Denkmalpflege zum Städtebau}

Besonders in seinen frühen Jahren zeigte sich Piacentini stark von den Ideen des malerischen Städtebaus beeindruckt, den Camillo Sitte um 1900 in Europa bekannt gemacht hatte. Sitte hatte beobachtet, dass historische Stadtplätze generell unregelmäßig und geschlossen angelegt waren, dass sie häufig in Gruppen auftraten und verschiedenen Funktionen zugeordnet waren. Zudem profitierten sie häufig von Aus- und Durchblicken sowie lokalen Besonderheiten. Die Prinzipien des Ortsbezugs, der Funktionsbezogenheit und der Geschlossenheit von Stadt- 
räumen nimmt Piacentini unmittelbar auf und macht sie zu Entwurfskriterien seiner eigenen Masterpläne. Das Beispiel der Piazza della Vittoria in Brescia zeigt, wie er sich darum bemüht, das historische Wegesystem durch die Implementierung eines neuen Platzes nach alten Regeln zu vervollständigen, um damit sowohl der alten Stadt als auch den neuen Bedürfnissen gerecht zu werden.

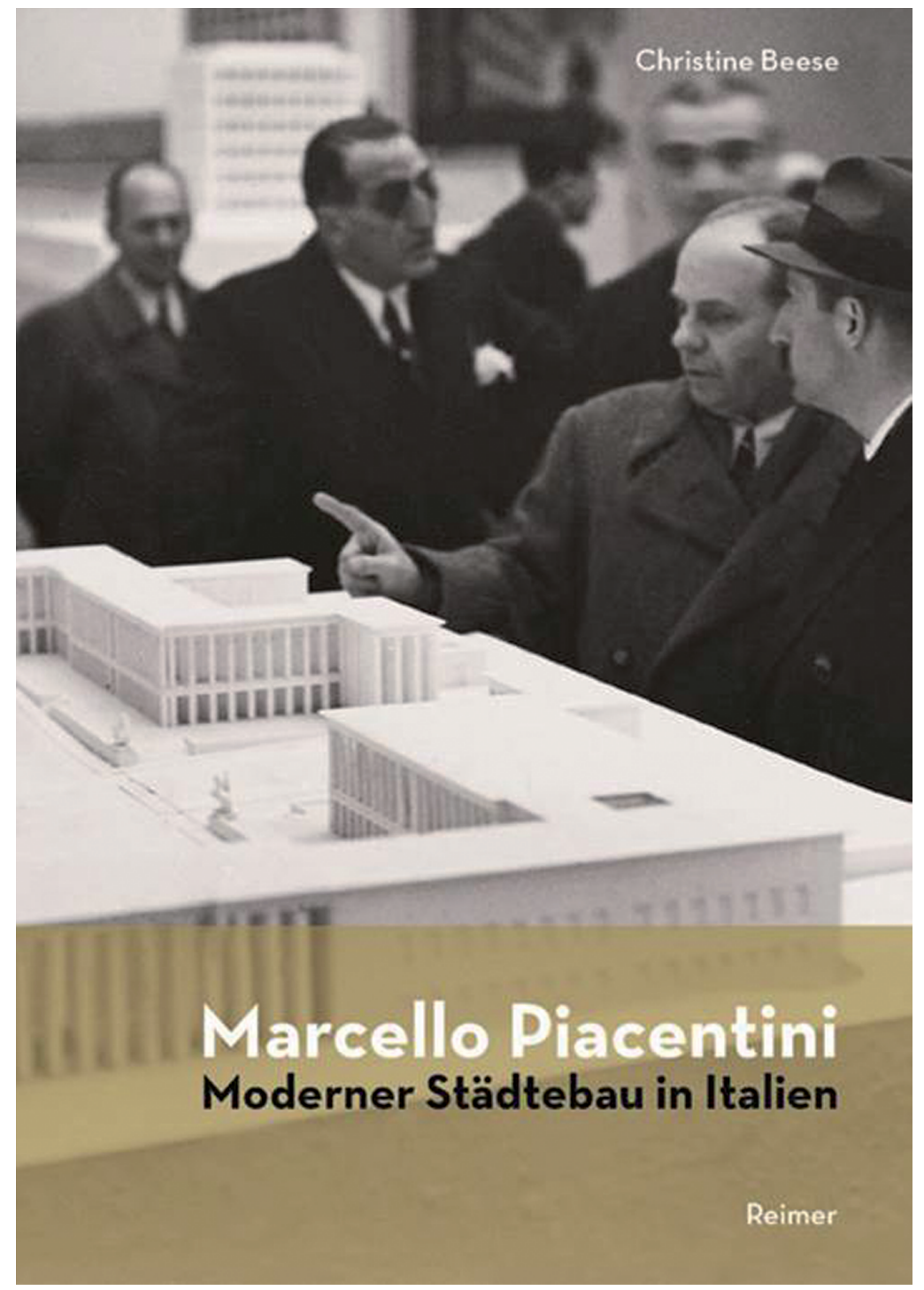

Abb. 5: Christine Beese: Marcello Piacentini. Moderner Städtebau in Italien, Berlin 2015. 


\section{Stadterweiterung - Rom als Metropole}

Piacentini war ein großer Bewunderer der europäischen Metropolen des 19. Jahrhunderts. Wien und Paris übten eine große Faszination auf ihn aus und sein Wunsch war es, Rom zu einer ebenso mondänen Weltstadt zu machen. Aus diesem Grund setzte er sich jahrzehntelang dafür ein, auf Höhe des heutigen Hauptbahnhofs ein neues, modernes Stadtzentrum zu schaffen, welches wie die Wiener Ringstraße oder die amerikanischen Civic Center Einrichtungen der Kultur, der Verwaltung und des geselligen Beisammenseins auf einem Platz vereinen sollte. Von der Verlagerung des modernen Lebens versprach er sich nicht nur den Erhalt der historischen Altstadt - die er einem solchen Publikumsverkehr nicht gewachsen sah - sondern auch konkrete Aufträge für sein wachsendes Architekturbüro.

Durch zahlreiche Reisen und internationale Zeitschriften war Piacentini mit den neuesten städtebaulichen Leitbildern in Europa vertraut. Besonders das Thema der Dezentralisierung beschäftigte in den 1920er Jahren die Fachwelt. Gemeinsam mit seinem Schüler Piccinato entwickelte auch Piacentini 1929 einen ersten Regionalplan für das Umland von Rom, dachte über das Verhältnis von Kernstadt, Stadterweiterung und Satellitenstädten nach. Im Gegensatz zum Denkmalpfleger Giovannoni wollte er nicht an der historischen Stadt als modernem Zentrum festhalten, im Gegensatz zu anderen Urbanisten sträubte er sich allerdings auch gegen reine Wohnsiedlungen außerhalb der Stadt. Sein Ziel blieb eine polyzentrische Stadterweiterung, wie er es aus Straßburg oder Nürnberg kannte.

\section{Plätze und Straßen - Bausteine einer Stadt}

Während die ersten beiden Kapitel meiner Arbeit chronologisch angelegt waren und Piacentinis Arbeit im Kontext der historischen Entwicklung untersucht haben, bedient sich das dritte Kapitel einem etwas anderen Zugriff. Hier ging es mir darum, anhand der Bauaufgabe Straße und Platz besondere Gestaltungsmerkmale herauszuarbeiten. Interessanterweise wurde hier deutlich, dass Piacentini zwar bestimmte Elemente wie Arkaden, Kolonnaden, Säulenportiken oder Türme immer wieder verwendet, dass er sich jedoch in Bezug auf die städtebauliche Anlage nicht festlegt. So finden sich auf der einen Seite Beispiele aus der Tradition des malerischen Städtebaus, wo Ausblicke inszeniert, Platzgruppen geschaffen und eine abwechslungsreiche Bebauung angestrebt wird. Auf der anderen Seite finden sich aber auch streng symmetrische und axiale Plätze, die der Beaux-ArtsTradition entspringen und mit einer einheitlichen Bebauung versehen sind. 


\section{Fazit}

Es lässt sich also festhalten, dass Piacentini nicht DEN einen faschistischen Städtebau entwickelt hat, sondern von Situation zu Situation verschiedene Grundformen gewählt hat. Zugleich bleibt er seiner Vorstellung von einem geschlossenen und räumlich erfahrbaren Stadtraum treu und widmet sich auch nach dem Krieg der Verbesserung innerstädtischer Situationen. Dass dabei immer wieder auch historische Bausubstanz verloren geht, nimmt er dann als Kollateralschaden in Kauf, wenn er der Ansicht ist, die bestehende Situation könne durch einen Eingriff verbessert werden. Im Gegensatz zu Le Corbusier denkt er nie daran, die historische Stadt einer völlig neuen Ordnung zu unterwerfen.

Durch seine Lehre als erster Städtebau-Dozent Italiens sowie durch die Beschäftigung der Studenten in seinem Büro hat Piacentini Generationen von jungen Architekten und Urbanisten geprägt. Seine Schüler besetzten die neuen Urbanistik-Lehrstühle im ganzen Land, sie gingen in Stadtplanungsämter oder eröffneten eigene Architekturbüros. Das besondere Verhältnis, welche italienische Architekten wie Giorgio Muratori oder Aldo Rossi zum gebauten Umfeld, zur räumlich geschlossenen Stadt entwickelten ist auch ein Erbe Marcello Piacentinis. 